\title{
Incubadora educacional: agregando tecnologias a prática do cuidado no ambiente de trabalho hospitalar
}

\section{Educational incubator: adding technologies to the practice of care in the hospital work environment}

\author{
Liliane Alves Pereira, Danielle Adriane Silveira Vidal, Cintia Marchesan Passos, Maria \\ Emília Bueno
}

Como citar este artigo: PEREIRA, L.A.; VIDAL, D. A. S.; PASSOS, C. M.; BUENO, M. E. N. Incubadora educacional: agregando tecnologias à prática do cuidado no ambiente de trabatho hospitalar. Revista Saúde (Sta. Maria). 2019; 45 (2)

\section{Autor correspondente:}

Nome: Liliane Alves Pereira

E-mail: irliliane7@hotmail.com

Telefone: (55) 98424-3685

Formação Profissional: Mestre em

enfermagem pela Universidade

Federal do Rio Grande (FURG),

Rio Grande, RS, Brasil.

Filiação Institucional: Universidade Franciscana

Endereço para correspondência: Rua: Silva Jardim n: 1670

Bairro: Centro Cidade: Santa Maria Estado: Rio Grande do Sul CEP: $97010-490$

Data de Submissão:

23/05/2018

Data de aceite:

27/07/2019

Conflito de Interesse: Não há conflito de interesse

\section{(c) $B Y-N C-N D$}

\section{RESUMO}

Objetivou-se conhecer as contribuições da Incubadora Educacional na perspectiva de novos colaboradores de enfermagem que participaram do processo de capacitação no ambiente de trabalho hospitalar. Métodos: pesquisa qualitativa, com 35 novos colaboradores sendo técnicos de enfermagem e enfermeiros, no período de julho de 2015 a julho de 2016, em um hospital de ensino da região sul do Brasil. A leitura dos dados foi realizada na perspectiva de Bardin. Resultados: Os colaboradores consideraram a incubadora educacional como adequada quanto a duração, conteúdo e método de ensino. Destacaram-se os núcleos temáticos: "A incubadora educacional como um processo de reciclagem" e "A integração entre teoria e prática no processo de aprendizagem". Conclusão: A Incubadora Educacional contribuiu de forma positiva no processo de capacitação dos novos colaboradores. Possibilitou um espaço de argumentações e reflexões sobre as práticas cotidianas da instituição envolvida e a participação ativa dos profissionais na busca por mudanças positivas na assistência.

PALAVRAS-CHAVE: Enfermagem; Aprendizagem; Inovações Tecnológicas.

\section{ABSTRACT}

Aimed to know the contributions of the Educational Incubator from the perspective of new nursing collaborators who participated in the training process in the hospital work environment. Methods: qualitative research, with 35 new collaborators being nursing technicians and nurses, from July 2015 to July 2016, in a teaching hospital in the southern region of Brazil. The data were read from Bardin's perspective. Results: The collaborators considered the educational incubator as adequate regarding duration, content and method of teaching. Thematic nuclei were highlighted: "The educational incubator as a recycling process" and "The integration between theory and practice in the learning process". Conclusion: The Educational Incubator contributed positively to the training process of the new collaborators. It provided a space for arguments and reflections on the daily practices of the institution involved and the active participation of professionals in the search for positive changes in care.

KEYWORDS: Nursing; Learning; Technological Development. 


\section{INTRODUÇÃO}

As tecnologias utilizadas para o aprendizado da enfermagem são compreendidas como a incorporação de ferramentas tecnológicas com fins pedagógicos, sendo realizadas tanto em ambientes de aprendizagem quanto de trabalho'. São capazes de qualificar a prática do ensino, em principal quando estão interligadas com o cotidiano do profissional de enfermagem.

Dentre essas tecnologias destaca-se a incubadora educacional que se realiza por meio de capacitações, sendo considerada uma nova forma de aprendizagem na enfermagem/saúde que permite integrar a inovação, a tecnologia e a educação. Possibilita aos trabalhadores da área captar a realidade do cotidiano, despertar e aplicar novas ideias, aumentar competências e habilidades, adquirir e adicionar conhecimento e, desta maneira, fazer diferença na sua trajetória profissional². Ela oferece orientações específicas para desenvolver serviços especializados.

A Incubadora Educacional ressalta aspectos voltados para a interação e a sensibilidade com o ambiente e o ser humano, oportunizando uma troca de experiências a partir da prática, buscando novas tecnologias e formas de cuidar². Vista nesta perspectiva, a Incubadora Educacional representa um novo modo de aprender, construindo uma aprendizagem integrada com a mudança/inovação, buscando criar e agregar tecnologias à prática do cuidado no ambiente de trabalho³. Este tipo de aprendizado é factível na medida que os colaboradores que fazem parte deste processo se tornam proativos do próprio processo de capacitação³.

Dentro da realidade da instituição hospitalar, onde os profissionais necessitam antever situações de risco, as formas de aprendizado precisam envolver questões do cotidiano da instituição, voltadas para as reais necessidades de tratamento e acompanhamento dos pacientes, visando a qualidade da assistência. A incubadora educacional possui um papel importante por integrar processos interativos e associativos de ensino-aprendizagem de acordo com as ações realizadas na prática assistencial ${ }^{4}$. Assim, o presente estudo teve como questão de pesquisa: quais são as contribuições da Incubadora Educacional para o processo de capacitação dos profissionais no ambiente de trabalho hospitalar? 0 objetivo foi conhecer as contribuições da Incubadora Educacional na perspectiva de novos colaboradores de enfermagem que participaram do processo de capacitação no ambiente de trabalho hospitalar.

\section{MÉTODOS}

Trata-se de uma pesquisa exploratória descritiva de abordagem qualitativa realizada em um hospital de ensino da região sul do Brasil. Fizeram parte do estudo 35 novos colaboradores da instituição contratados para fazer parte do 
corpo da equipe de Enfermagem, compreendidos entre técnicos de enfermagem e enfermeiros, no período de julho de 2015 a julho de 2016. Foram considerados como critérios de inclusão o ingresso na instituição neste período e ter participado da Incubadora Educacional.

O projeto iniciou com a finalidade de promover o acolhimento e a integração do funcionário recém admitido na Instituição; qualificar o trabalho desenvolvido pela equipe de enfermagem; Identificar a percepção do novo colaborador sobre o ingresso na Instituição; Identificar barreiras e facilidades no processo de adaptação do colaborador que participa da Incubadora e propor melhorias neste processo.

As atividades da Incubadora Educacional foram realizadas pelo serviço de educação permanente do hospital e aconteceram a partir do momento do contrato dos novos colaboradores, com seguimento durante as primeiras quatro semanas de inserção do colaborador na Instituição.

Esse recurso facilitou o acompanhamento e as transformações constantes e inevitáveis que norteiam a inserção do novo colaborador na estrutura do trabalho. Os colaboradores foram previamente comunicados através de convocações, as quais foram identificadas com a data, o local e a hora e aconteceram durante o expediente, nos turnos da manhã, tarde e noite.

Ao final das capacitações, foi aplicado um questionário constituído de perguntas fechadas e abertas compondo a percepção acerca das facilidades e barreiras para o processo de capacitação e adaptação à instituição. A entrevista teve como questões norteadoras as expectativas que o novo colaborador tinha ao entrar na instituição, as dificuldades e facilidades no processo de adaptação na unidade e com a equipe de trabalho. Nas questões fechadas foi utilizada duas opções de respostas: "adequada" e "inadequada" para avaliar as diferentes etapas da pesquisa que são descritas a seguir.

Os dados obtidos foram tratadas de forma ética e fielmente transcritas, sem interferência de pré-julgamentos, garantindo o anonimato e respeitando todos os preceitos éticos e legais que regem a pesquisa com seres humanos, estabelecidos pela Resolução 466/12 do Conselho Nacional de Saúde ${ }^{5}$. O estudo recebeu uma certificação ética sob o número 05/2015. 0 estudo recebeu parecer somente pelo comitê de ética da instituição vinculada à pesquisa.

A leitura dos dados foi realizada mediante análise de conteúdo temática, que consiste nas seguintes etapas: $\mathrm{A}$ primeira, denominada pré-análise, na qual foi realizada a leitura exaustiva dos dados, seguida da organização do material e formulação de hipóteses. A seguir, foi realizada a exploração do material, ou seja, os dados brutos foram codificados e a terceira e última etapa, consistiu na interpretação e delimitação dos dados em categorias temáticas pela compreensão dos significados ${ }^{6}$. 


\section{RESULTADOS}

Na primeira etapa de capacitação foi realizada uma integração, com a apresentação da instituição, bem como demonstração do organograma, fluxos e serviços de apoio. Na segunda etapa foi feita a descrição e apresentação dos principais formulários de registros, pedidos e encaminhamentos utilizados pela equipe de enfermagem, além dos Procedimentos Operacionais Padrão (POPs), manuais, regimentos e regulamentos. Estas ações buscam incentivar a procura do conhecimento institucional por parte do funcionário.

Posteriormente, abordaram-se assuntos relativos ao controle de infecção, utilização dos equipamentos de proteção individual (EPIs) e condutas adequadas no manejo de pacientes em isolamento. Na última etapa foi realizada uma revisão da assistência de enfermagem ao paciente e uma avaliação formal dos funcionários e da Incubadora Educacional. Tal avaliação aconteceu por meio de um questionário com questões abertas e fechadas, o qual deu origem à esta pesquisa.

Cabe salientar que uma etapa é independente da outra, o que facilita no momento de acolhimento, já que devido ao período de contratações estarem ocorrendo concomitante com as capacitações, os funcionários participam de maneira que complete as quatro etapas, independente da ordem e do período em que o mesmo estiver iniciando na Instituição.

Dentre os 35 novos colaboradores que participaram das capacitações, nove (26\%) foram enfermeiros e 26 (74\%) técnicos de enfermagem, com idades entre 24 a 45 anos. Todos eles consideraram as quatro etapas da incubadora educacional como adequada quanto a duração, conteúdo e método de ensino. Após a análise dos dados, a avaliação do processo de capacitação foi representada por dois núcleos temáticos, conforme mostrado na tabela 1.

Tabela 1 - Avaliação do processo de capacitação

\begin{tabular}{|l|l|}
\hline \multicolumn{1}{|c|}{ Núcleos temáticos } & \multicolumn{1}{c|}{ Unidades de Registro } \\
\hline $\begin{array}{l}\text { A incubadora educacional como } \\
\text { um processo de reciclagem e e } \\
\text { atualização }\end{array}$ & $\begin{array}{l}\text { Retomada de conteúdos vistos no início do processo de formação, auxiliando } \\
\text { no desempenho profissional; } \\
\text { Segurança na realização das ações de cuidado; } \\
\text { Aprendizado sobre a assistência de enfermagem como um todo; } \\
\text { Conhecimento das rotinas de cada setor na instituição. } \\
\text { Conhecimento global da instituição; }\end{array}$ \\
\hline $\begin{array}{l}\text { A integração entre teoria e prática } \\
\text { no processo de aprendizagem }\end{array}$ & $\begin{array}{l}\text { Possibilidade de reflexão sobre as ações do cuidado; } \\
\text { Crescimento pessoal e profissional; } \\
\text { Facilidade de adaptação na instituição; }\end{array}$ \\
\hline
\end{tabular}

Fonte: Dados da pesquisa, 2016. 
O núcleo temático "A incubadora educacional como um processo de reciclagem e atualização" agrupou aspectos que estavam relacionados com a capacidade de aperfeiçoamento e aprimoramento profissional. Os profissionais perceberam que, após a realização das capacitações, as ações de cuidado foram desenvolvidas com mais segurança, obtiveram maior facilidade de adaptação no serviço e foram capazes também de conhecer e se adaptarem às rotinas de cada setor com maior facilidade, obtendo com isso, um conhecimento global da instituição. A retomada de alguns conteúdos vistos no processo de formação profissional foi outro aspecto considerado pelos novos colaboradores como fundamental para o desempenho das ações assistenciais.

A integração entre teoria e prática no processo de aprendizagem foi o segundo núcleo temático que agrupou as seguintes unidades de registro: Possibilidade de reflexão sobre as ações do cuidado; Crescimento pessoal e profissional; Facilidade de adaptação na instituição. Os participantes relataram que esse encontro propiciou um novo olhar acerca da instituição. Percebeu-se que após esse encontro os funcionários puderam desenvolver empatia em relação ao serviço se seus colegas, o que colaborou para o trabalho da equipe multidisciplinar.

Isso permitiu também o aprimoramento da capacidade de reflexão sobre as ações de cuidado do paciente, possibilitando a compreensão do ato do "fazer". Este dinamismo proporcionou segurança e autonomia para os novos colaboradores, até mesmo para interagirem com os demais membros da equipe de trabalho com mais facilidade, proporcionando uma melhor adaptação em um menor espaço de tempo na instituição.

\section{DISCUSSÃO}

Os resultados do estudo permitiram constatar que as capacitações realizadas por meio da Incubadora Educacional ofertaram um conhecimento global da instituição para os novos colaboradores, bem como a contribuição para o desenvolvimento efetivo da prática assistencial. Os participantes conseguiram compreender que a proposta de capacitação estava associada com a realidade de trabalho da instituição a qual estão inseridos, bem como a importância do embasamento teórico para o desempenho das ações de cuidado. Houve valorização da teoria para agregar novos conhecimentos e desempenhar uma prática efetiva. Esta posição é corroborada por estudo realizado com profissionais da enfermagem em um hospital universitário em que se destaca a aquisição do conhecimento científico para orientar e padronizar as ações de cuidado ${ }^{7}$.

As capacitações articuladas com a realidade institucional tem a perspectiva de utilizar métodos participativos em que os colaboradores possam ter autonomia e percebam as potencialidades e limitações de sua prática ${ }^{8}$. Foi possível observar que esta percepção foi acontecendo de forma gradual com os novos colaboradores deste estudo, aumentando 
as expectativas e as necessidades de aprimoramento profissional. De acordo com alguns autores, o processo de capacitações de colaboradores de saúde precisa estar estruturado a partir da problematização do processo de trabalho, considerando as necessidades de saúde das pessoas e da população9,10.

Neste sentido, a implantação e desenvolvimento da incubadora educacional voltada para a realidade do local de trabalho possibilitou focar a prática como fonte de conhecimento e estimular os colaboradores a atuarem ativamente no processo educativo. Estudo que buscou descrever as práticas educativas em saúde para os profissionais de enfermagem aponta que a educação surge como ferramenta capaz de despertar no trabalhador o desejo de discutir e refletir acerca do processo de trabalho ${ }^{11}$.

A facilidade de engajamento na equipe de trabalho e a reflexão sobre as ações na prática assistencial também foram destacados como aspectos positivos. Tais constatações foram semelhantes ao de uma pesquisa realizada com 34 colaboradores de um hospital de ensino da região central do Rio Grande do Sul, a qual aponta que a incubadora possibilita aos colaboradores conhecer colegas de diferentes setores e a capacidade de refletir sobre sua prática ${ }^{4}$. Pesquisa que apontou a incubadora educacional como uma ferramenta para intervir e (re)pensar os cuidados de saúde também constatou que há uma maior interação entre os colaboradores, além da melhoria na comunicação e ação assistencial $^{12}$.

O estímulo ao aprendizado suscitou nos novos colaboradores a visão de desenvolvimento pessoal e profissional. Este tipo de sentimento gera uma capacidade maior de satisfação no desempenho de suas ações no ambiente de trabalho. Estudo que buscou conhecer as contribuições deste tipo de trabalho para os colaboradores encontrou resultado semelhante ao inferir que neste processo de aprendizado é possível transcender e ampliar as possibilidades pessoais e coletivas ${ }^{4}$. Sendo assim, a incubadora educacional possibilita (re)pensar atitudes e condutas no intuito de ampliar 0 cuidado/assistência pontual, mecanicista e linear ${ }^{12}$.

A oportunidade de participar da Incubadora Educacional possibilitou aos colaboradores a discussão de problemas cotidianos dos setores, permitindo assim, a percepção destes como parte do processo de trabalho. Esta concepção faz com que haja um posicionamento mais ativo e participativo por parte dos novos colaboradores no ambiente de trabalho. Esta inovação é a base para que a Incubadora Educacional seja uma forma de renovar os profissionais e os tornar cada vez mais aptos para um cuidado ético. Promove também uma auto valorização por parte dos colaboradores de enfermagem, propiciando segurança para as ações do cuidado e construindo uma mudança positiva na prática assistencial13,14.

Com tais benefícios foi possível perceber que o presente estudo apresentou como limite o fato de a incubadora educacional ter sido realizada somente com os novos colaboradores, não incluindo nas capacitações os trabalhadores mais antigos na instituição. Desta forma, permanece a necessidade de que os profissionais de saúde estejam engajados 
na busca da participação e organização ativa de capacitações, na busca de conhecimento e das possibilidades de sua aplicação na prática.

\section{CONSIDERAÇÕES FINAIS}

A Incubadora Educacional contribuiu de forma positiva no processo de capacitação dos novos colaboradores da equipe de enfermagem. Possibilitou um espaço de argumentações e reflexões sobre as práticas cotidianas da Instituição envolvida, bem como a participação ativa dos profissionais na busca por mudanças que venham a contribuir de forma positiva para a assistência do paciente. Tais mudanças estão relacionadas desde a postura profissional frente às situações que envolvem o cotidiano da prática, quanto a conscientização da realização de ações voltadas para a diminuição da exposição do paciente aos riscos provenientes da intervenção de enfermagem.

A possibilidade de refletir sobre as ações de cuidado prestado ao paciente, instiga mudanças efetivas capazes de implementar novos recursos e possibilidades de desenvolver a prática assistencial e torna a Incubadora Educacional uma ferramenta importante para o trabalho de enfermagem e o aperfeiçoamento profissional. Por meio deste processo de capacitação é possível tornar o profissional protagonista de suas ações assistenciais, permitindo-o refletir sobre 0 fazer cotidiano e mecanizado e abrindo novas possibilidades de inovação, embasadas nas necessidades individuais e institucionais. Desta forma, é possível construir uma enfermagem cada vez mais científica, tecnológica e autônoma, que desde a sua inserção no mercado de trabalho reflete sobre suas ações e presta um cuidado científico, ético, estético e seguro aqueles que lhes são confiados.

\section{REFERÊNCIAS}

1. Salvador PTC, Rodrigues CCF, Lima KYN, Alves KYA, Santos VEP. Uso e desenvolvimento de tecnologias para o ensino apresentados em pesquisas de enfermagem. Rev Rene. 2015 maio-jun; 16(3):442-50.

2. Cecagno D, Siqueira HCH, Calvetti A, Castro QL, Erdmann AL. Incubadora de aprendizagem na enfermagem: inovação no ensino do cuidado. Rev. Bras. Enferm. 2009 maio-jun; 62(3):463-6.

3. Gonçalves MFC, Andrade LS. Ensinar e aprender saúde: processos de aprendizagem e o uso da tecnologia. Sal.\&Transf. Soc. 2016; 7(2):1-10. 
4. Backes DS, Obem MK, Pereira SB, Gomes CA, Backes MTS, Erdmann AL. Incubadora de Aprendizagem: ferramenta indutora do empreendedorismo na Enfermagem. Rev. Bras. Enferm. 2015; 68(6):1103-08.

5. Brasil. Ministério da Saúde. Conselho Nacional de Saúde. Resolução $n^{0} 466$, de 12 de dezembro de 2012. Aprova as diretrizes e normas regulamentadoras de pesquisas envolvendo seres humanos. Diário Oficial da União, Brasilia, 13 jun. 2013, p.59.

6. Bardin L. Análise de conteúdo. Lisboa, Portugal: Edições 70; 2011.

7. Jesus MCP, Figueiredo MAG, Santos SMR, Amaral AMM, Rocha LO, Thiollent MJM. Educação permanente em enfermagem em um hospital universitário. Rev. esc. enferm. USP. 2011 Oct;45(5):1229-36.

8. Miccas FL, Batista SHSS. Educação permanente em saúde: metassíntese. Rev. Saúde Pública. 2014; 48(1):170-185.

9. Barbosa NCT, Cordeiro CB. The permanent education in health: an analysis of intervention projects submitted by managers of health during the participation of the course of micropolitical (ead): prior note. Journal of Nursing UFPE. 2016; 10(5):1895-1898.

10. Giovana Ely Flores GE, Oliveira DLL, Zocche DAA. Educação permanente no contexto hospitalar: a experiência que ressignifica o cuidado em enfermagem. Trab. Educ. Saúde, Rio de Janeiro. 2016; 14(2):487-504.

11. Salum NC, Prado ML. Continuing education in the development of competences in nurses. Texto Contexto Enferm. 2014; 23(2):301-8.

12. Mello AL, Backes DS, Terra MG, Rangel RF, Nietsche EA, Salbego C. (Re) pensando a educação permanente com base em novas metodologias de intervenção em saúde. Revista Cubana de Enfermería. 2017; 33(3).

13. Lubejko BG. Developing a Program Evaluation Plan: Options and Opportunities.J Contin. Educ. Nurs. 2016; 47(9):388-389. 
14. Pereira FM, Barbosa VBA, Vernasque JRS. Continuing education experience for auxiliary nurses as a management strategy. REME Rev Min Enferm. 2014; 18(1):228-35. 